\title{
Knowledge, Attitude and Practice of Exclusive Breastfeeding Among Mothers Attending an Infant Welfare Clinic in Osogbo, Osun State, Nigeria
}

\author{
Sola Odu, Samson Ayo Deji", Eyitope Amu, Victor Aduayi \\ Department of Epidemiology and Community Health, Faculty of Clinical Sciences, College of Medicine, Ekiti State University, Ado Ekiti, \\ Nigeria
}

\author{
Email address: \\ samdeji3@hotmail.com (S. A. Deji) \\ ${ }^{*}$ Corresponding author
}

\section{To cite this article:}

Sola Odu, Samson Ayo Deji, Eyitope Amu, Victor Aduayi. Knowledge, Attitude and Practice of Exclusive Breastfeeding Among Mothers Attending an Infant Welfare Clinic in Osogbo, Osun State, Nigeria. European Journal of Preventive Medicine. Vol. 4, No. 2, 2016 , pp. $39-43$. doi: $10.11648 /$ j.ejpm.20160402.13

Received: January 26, 2016; Accepted: February 16, 2016; Published: March 23, 2016

\begin{abstract}
There is much concerns that despite the "Innocenti Declaration" and all efforts to promote Exclusive Breastfeeding in Nigeria, the prevalence of malnutrition and infant mortality is high. The study determined the knowledge, attitudes and practices (KAP) of nursing mothers in Osogbo, Nigeria. The study design was cross sectional. A total of 328 nursing mothers attending infant welfare clinics were recruited from selected four health centres in Osogbo Local Government Area by convenience sampling method. Semi-structured questionnaires were used to collect data on the Knowledge, Attitudes and Practices of respondents. Data were analyzed using Statistics Package for Social Scientists (SPSS) version 16. About $97.6 \%$ of the respondents were aware of EBF, but only $64.6 \%$ had adequate knowledge. Majority of the respondents (92.7\%) learnt about EBF from health workers. Attitude to EBF was good as reported by $75.6 \%$ of respondents who practiced EBF on demand. About $73.8 \%$ of respondents practiced EBF. The respondents have good knowledge and attitude of EBF. The practice of EBF was equally good however less than one third used either water or herbs during EBF before six months.
\end{abstract}

Keywords: Exclusive, Breastfeeding, Knowledge, Practices, Mothers

\section{Introduction}

Breastfeeding is the means by which nutrition is provided, for the healthy growth and development of infants by putting the nipple of the mother's breast into the mouth of the baby. [1] It is also an integral part of the reproductive process with important implications for the health of mothers. [2] Breastfeeding contributes to the health and wellbeing of mothers, helps to space children, reduces the risk of ovarian and of breast cancers and increases family and natural resources. [1] It is a secured way of feeding infants and it is safe for the environment. The balance of nutrients and chemical constituents in breast milk from a healthy mother makes it ideal for young infants. [2] It provides all the energy and nutrients that the infant needs in the first few months of life and continues to provide about half of a child's nutritional needs during the second half of the first year and about one-third during the second year of life. [3] Breast milk promotes sensory and cognitive development of the child and protects it against infections and chronic diseases. [4] Breastfeeding is generally accepted globally by all cultures as the mode of feeding the newborn but the problems have always been how well it is done to improve the health of the infants. [3-4]

Exclusive breastfeeding (EBF) is defined as the practice of giving no other food or drink except breast milk for the first six months of life to the newborn. [3] "Predominant breastfeeding" means that the infant's predominant source of nourishment is the breast milk. [3] Exclusive breast feeding reduces infant mortality due to common childhood illness such as diarrhea and helps for quicker recovery during illness. [5-8] There is no added advantage in supplementing the human breast milk with solids food before six months of age for growing infants. [2] In other words, the nutritional 
needs of the neonates could be adequately met by giving breast milk alone.

In Nigeria, many changes in infant feeding practices have occurred over time, due to the introduction of alien cultures and values, urbanization with consequent changes in lifestyle and adoption of foreign practices by members of the society. All these have affected the practice of breastfeeding and use of breast milk substitutes. [9]

In Europe and America artificial feeding using cow's milk was not even mentioned as a substitute for breastfeeding until the eighteenth century. [10] Few infants, if any survived unless they were breast fed. When the mother was unable to breastfeed, the only alternative was the employment of a wet nurse. The practice continued in Europe till the end of the nineteenth century when as result of several abuses on the part of the wet nurses, they became unpopular. [11]

While breastfeeding is a natural act, it is also a learned behavior. Breastfeeding of new born infants may be influenced by a number of factors including the socioeconomic status of mothers, variation in health status of mothers, working class mothers, cultural values and beliefs of the people among others. [8-9] Breastfeeding has considerable advantages over artificial feeding of babies under six months. [10-13] Studies carried out in Enugu, a city located in eastern part of Nigeria, revealed the exclusive breastfeeding rate to be $33.3 \%$. [14] Likewise, studies carried out in Ibadan, Nigeria revealed that EBF rate dropped from $57.4 \%$ at one month to $23.4 \%$ at six months. [15] These low rates of EBF account for the increase in infant morbidity and mortality which result from varying severity of gastroenteritis and malnutrition.

Knowledge determines the attitude and the attitude of the people in turn dictates their practices. How much people know about the maintenance of breastfeeding will go a long way in influencing their input and practice to ensure successful breastfeeding. [16] This study was therefore conducted to determine the knowledge, attitude and practice of EBF among mothers of infants attending infants Welfare Clinics in Osogbo Local Government Area (OSLGA) of Osun State, Nigeria.

\section{Materials and Methods}

A cross sectional descriptive study design was employed in conducting the survey. The study was conducted at Osogbo Local government area (LGA) in Osun State located in south western Nigeria.

Osogbo local government is well supplied by the natural grid, it is predominantly a low land area with rocky subterranean terrainin certain areas like Oke-Baale, OkeAyepe, Oke-Osun and Agunbelewo -the vegetation is mainly savannah. Residents of the area are predominantly Yoruba, with Hausa and Ibo settlers.

Majority of the people are either Christians or Muslims. Prominent occupations embraced are farming, weaving, trading and art work (dyeing and painting). Traditionally they observe the Osun State Osogbo festival yearly.
The local government is divided into 15 electoral wards. The area is urbanized with social facilities like tertiary institutions eg Ladoke Akintola University Teaching Hospital, public and private primary and secondary schools, one technical and nursing school, banks, hostel, markets among others.

There are 9 primary health centers spread across the local government wards; while 6 serve the urban community, 3 serve the rural areas. The instrument of study was a pretested semi structured questionnaire in four parts covering the entire scope of the study such as the socio-demographic data, the knowledge, attitude and practice (KAP) of respondents with respect to $\mathrm{EBF}$.

Three hundred and twenty eight nursing mothers were recruited from selected health centres located at the study areas of the Local Government Area. (LGA) The respondents were recruited to the study by convenience sampling method. The health centres were sorted according to their locations into rural or urban. Majority of the population in the LGA lived in the urban areas compared to those in rural area. Four health centres from the urban area and one from the rural area were randomly selected by balloting representing the entire LGA.

The infant welfare clinic held once a week [every Tuesday] in the entire health centres of the local government area.

Using the modified Leslie Fisher's formula for single proportions a sample size of 328 was determined.

Ethical clearance was obtained from the Research and Ethical Committee of Ladoke Akintola University, Ogbomoso, Nigeria. The respondents' consent was gained verbally and confidentiality was assured. Their participation in the study was entirely voluntary. However, those who do not meet the criteria for the study such as caregivers who are not direct mothers to the children were exempted from the study.

Measurements of variables.

Knowledge was measured using a set of questions on EBF especially on the durations and the point of initiation of breastfeeding. Respondents who answered positively were assessed to be knowledgeable about EBF.

Attitude was measured using a set of questions that bothered on the reactions and fulfillments of mothers during $E B F$. Respondents who answer in the affirmative were scored to have had positive attitude.

Practice was measured by assessing responses to questions that bothered on the conduct of EBF. Those with positive responses were assumed to have had good practices of $E B F$

Quantitative data was collected on variables such as the socio-demographic characteristics, knowledge of the respondents on EBF, their attitude and practices of EBF. Data was collected from 328 nursing mothers attending the clinics using an interviewer administered questionnaires. Data was analyzed using software of Statistics Package for Social Sciences (SPSS) version 16. The level of statistical significance was set at $<0.05$. 


\section{Results}

Table 1 shows that the majority $(90.3 \%)$ of respondent were within $27-30$ years age range. All respondents had one form of education or the other with $66.5 \%$ having had secondary level education. Most respondents, $44.5 \%$, were traders.

Table 2 shows $97.6 \%$ of the respondents claimed to have knowledge about EBF though a lesser percentage (64.6\%) had adequate knowledge. About $75 \%$ knew that EBF should be initiated immediately after birth; $82.3 \%$ knew that EBF should be given on demand by the baby, while $73.8 \%$ thought that herbs should be given during EBF and 35.4\% did not give correct response to duration of $\mathrm{EBF}, 92.7 \%$ of respondents learnt EBF from health workers.

Table 3 shows the majority of respondent (78\%) felt fulfilled after EBF, while $96.3 \%$ believed that EBF is beneficial to the infants under six months of age.

Table 4 shows $93.3 \%$ of the respondent claimed they had adequate spousal support when observing EBF; 68.3\% continued EBF even when ill and $75.6 \%$ practiced EBF on demand by the babies. A good number, $73.8 \%$, introduced supplementary feeds after 6 months.

Table 1. Sociodemographic characteristics of respondents.

\begin{tabular}{|c|c|c|}
\hline Variable & Frequency $\mathbf{N}=\mathbf{3 2 8}$ & $\%$ \\
\hline \multicolumn{3}{|l|}{ Age } \\
\hline$<20$ years & 22 & 6.70 \\
\hline $20-29$ years & 182 & 55.49 \\
\hline $30-39$ years & 114 & 34.76 \\
\hline \multicolumn{2}{|l|}{ Occupation } & 3.05 \\
\hline Civil Servant & 20 & 6.10 \\
\hline Traders & 146 & 44.50 \\
\hline Students & 24 & 7.30 \\
\hline \multicolumn{3}{|l|}{ Ethnic Group } \\
\hline Yoruba & 320 & 97.60 \\
\hline Ibo & 6 & 1.80 \\
\hline Others & 2 & 0.60 \\
\hline \multicolumn{3}{|l|}{ Educational Status } \\
\hline Primary level & 60 & 18.30 \\
\hline Secondary level & 218 & 66.50 \\
\hline Tertiary level & 50 & 15.20 \\
\hline
\end{tabular}

Table 2. Respondents' Knowledge on EBF with respect to normal duration of EBF, source of information, Initiation, Frequency and Additional Feeding.

\begin{tabular}{lll}
\hline Variable & Frequency $\mathbf{N = 3 2 8}$ & $\mathbf{\%}$ \\
\hline Duration of EBF & & \\
1 month & 4 & 1.20 \\
$2-3$ months & 6 & 1.80 \\
$4-6$ Months & 212 & 64.60 \\
$>6$ Months & 106 & 32.40 \\
Source of Knowledge of EBF & & \\
Health Workers & 304 & 92.70 \\
Family & 6 & 1.80 \\
Mass Media & 18 & 5.50 \\
When to initiate EBF & & \\
Immediately after delivery & 246 & 75.00 \\
\hline
\end{tabular}

\begin{tabular}{lll}
\hline Variable & Frequency $\mathbf{N = 3 2 8}$ & $\mathbf{\%}$ \\
\hline Few hours after delivery & 62 & 19.00 \\
Few days after delivery & 10 & 3.00 \\
Don't know & 10 & 3.00 \\
$\begin{array}{l}\text { Frequency of Episode EBF } \\
\text { On Demand }\end{array}$ & 270 & \\
Every Hour & 44 & 82.30 \\
$\begin{array}{l}\text { Don't Know } \\
\text { Do you thinkherbs should be added } \\
\text { while practicing EBF. }\end{array}$ & 14 & 13.40 \\
Yes & 86 & 4.30 \\
No & 242 & \\
\hline
\end{tabular}

Table 3. Respondents' Attitude to EBF.

\begin{tabular}{|c|c|c|}
\hline Variable & Frequency & $\%$ \\
\hline \multicolumn{3}{|l|}{ Does child's crying indicate that you give water } \\
\hline Yes & 108 & 32.90 \\
\hline No & 220 & 67.10 \\
\hline \multicolumn{3}{|l|}{ Mother feeling after EBF } \\
\hline Fulfilled & 256 & 78.00 \\
\hline Compulsory & 6 & 1.80 \\
\hline Tasking & 44 & 13.40 \\
\hline Don’t Know & 22 & 6.70 \\
\hline \multicolumn{3}{|l|}{$\begin{array}{l}\text { Is EBF beneficial to infants under } 6 \text { months of } \\
\text { age? }\end{array}$} \\
\hline Yes & 316 & 96.30 \\
\hline No & 12 & 36.70 \\
\hline Total & 328 & 100 \\
\hline
\end{tabular}

Table 4. Respondents' Practice of EBF.

\begin{tabular}{lll}
\hline Variable & Frequency $\mathbf{N}=\mathbf{3 2 8}$ & $\mathbf{\%}$ \\
\hline Spousal Support of EBF & & \\
Yes & 306 & 93.3 \\
No & 22 & 6.7 \\
Does your being ill stop EBF? & & \\
Yes & 104 & 31.7 \\
No & 224 & 68.3 \\
Exclusive BF on demand & & \\
Yes & 248 & 75.6 \\
No & 80 & 24.4 \\
Duration of each episode of EBF & & \\
Until child is satisfied & 202 & 79.9 \\
$5-20$ mins & 28 & 8.5 \\
$>20$ mins & 38 & 11.6 \\
Introduction of supplementary feeds & & 3.0 \\
From birth & 10 & 4.3 \\
At 3 Months & 14 & 18.9 \\
$4-6$ Months & 62 & 73.8 \\
$>6$ Months & 242 & \\
Do you give herbs or concoction during & & 21.3 \\
EBF & & 78.7 \\
Yes & 70 & \\
No & 208 & \\
\hline & & \\
\hline & &
\end{tabular}

\section{Discussion}

The study set to assess the knowledge, attitude and practices of mothers attending infant Welfare Clinics in Osogbo Local Government Area of Osun State Nigeria.

Majority $(90.25 \%)$ of the respondents were within the age range of 20 and 39 years with a mean age of 26 years. This revealed that most of the mothers were matured women and implied that child marriages were uncommon in the study area. Almost all respondents had one form of education or the other 
which could have accounted for delay in the age before marriage. This is in consonance with a previous study carried out in Sub-Saharan Africa which showed that the average age at marriage increased with educational level. [16]

This study reported $64.6 \%$ of respondents had knowledge that EBF should continue until 6 months. This is not in consonance with a study done in Ethiopia where it was reported that $83.4 \%$ of mothers were knowledgeable about the recommended duration of EBF. [18] It should have been expected that a higher percentage of the respondents should have a much more higher percentage of knowledge with respect to the correct period for EBF as demonstrated by the study in Ethiopia. [17] The varied knowledge is common in different parts of African countries due to several factors especially the literacy levels and the accessibility to information desks on health matters.

About $75 \%$ of respondents reported to have started breastfeeding immediately after delivery of their babies less than 30 minutes which is the recommended guidelines for breastfeeding by World Health Organization (WHO). [18] This is a good index of EBF in the study areas, but it should have been expected that a greater percentage of the respondents complied with the WHO recommended guideline because the introduction of breastfeeding within the first few hours of life is very crucial to the development and growth of the children.

About $75.6 \%$ of mothers reported to have practiced EBF in the study areas. However, $73.8 \%$ of respondents reported commencing additional complementary meals to their children after age 6 months. This is contrary to a study done in Bangladesh which reported that only $31.0 \%$ children were exclusively breastfed for the first 6 months of life. [19] The presume positive practice could not be unconnected to the fact that the respondents were literate and have learnt of the health benefits of EBF from health workers which has resulted to positive behavioural changes. This is also contrary to a study conducted in Calabar, Nigeria, which revealed the present EBF rate to be $22.9 \%$ among nursing mothers. [20] Most places in developing countries have low rate of the practice of EBF. Among many factors that are generally responsible for the low practice of EBF is illiteracy and lack of adequate awareness among nursing mothers on the importance of total compliance with EBF in the first six months of life for their babies.

About one-quarter of respondents reported that they stopped EBF when the child took ill and gave herbs or concoctions as treatment options. These are some of the practices in most developing countries especially where they uphold traditional beliefs of using herbal medicine to cure illnesses instead of orthodox medicine. These accounted for some drawbacks in the practice of EBF in the study areas. The traditional practices of women giving native concoctions reported in the study area is in keeping with a study carried out in Igbo Ora Nigeria where respondents were also in the habit of giving herbal medicines and introduction of water to their children whenever they took ill without due consultation of medical personnel in approved health facility centre sat their jurisdiction. [21]

However it is worthy of note that a good percentage of respondents, $75.6 \%$ fed their children with EBF on demand which is a good healthy practice of infant feeding. This is against the backdrop that some women held the opinion that breastfeeding on demand is not a modern method of infant breastfeeding, hence there is early introduction of complementary feeding and forced feeding of children with feeding bottles. [1] This however again cannot be unconnected with the level of literacy of such nursing mothers. A well informed woman will naturally know the importance of giving EBF to their babies on demand because it will enhance the growth and development of such children and reduce the level of excessive cries due to hunger..

Spouses' supports may also have contributed to the positive practices of EBF reported in the study area. Over $90 \%$ reported that they had their spouse support while observing EBF. The support may simply mean helping the woman to do other domestic cores that could distract her attention from concentrating on breastfeeding the baby. In most African countries, the tight schedule of the men most often may not allow them to stay with their wives while breastfeeding during the first few months of life of the baby. [22] A good proportion of the respondents were literate and most likely had their spouses as literates who understood the importance of breastfeeding in the first few months of life. Moral support and encouragements from such husbands would have gone a long a way to increase EBF compliance among nursing mothers in the study areas.

\section{Conclusion}

The knowledge, attitude and practices of nursing mothers with respect to EBF was found to be high. In this study, two thirds of mothers practiced exclusive breastfeeding.

The respondents have good knowledge and attitude, but the practice of EBF was not as expected because some of them gave water or herbs during EBF periods while others stopped EBF before 6months which is the recommended time frame for $E B F$.

\section{Recommendations}

There is therefore a need to intensify health education on the benefits and management of breastfeeding. Health workers should receive more training concerning dissemination of information on EBF. Creches should be made available for public workers to enhance exclusive breastfeeding. Good motherhood and breast feeding practices should be re-awakened and encouraged through mass campaigns.

Improving access to information on recommended infant feeding practices during routine maternal and child health services is recommended. It is important to encourage EBF among the young, employed and urban mothers through proper counseling and mother friendly work environment.

Education of women on motherhood should be 
incorporated into social studies from secondary institution.

\section{References}

[1] M N Ike. Utilization of Exclusive Breast Feeding Methods among Nursing Mothers in Nigeria. Mediterranean Journal of Scocial Sciences MCSER Publishing, Rome Italy. 2013; Doi. 5901; 4(8).

[2] www.who.int/child-adolescenthealth/Nutrition/Infant_exclusive.htmaccessed in 2015.

[3] Azubuike JC, Nkanginieme KEO. Paediatrics and Child Health in a Tropic Region. Second Edition African Educational Services Owerri, Nigeria 2007; 165-167.

[4] www.who.int/nut/db-bfd.htm W.H.O Global Data Bank on Breastfeeding accessed in 2015.

[5] Countsoudis, A. et. al. 1998. Influence of infant feeding patterns on early mother - to- child transmission of HIV one in Durhan. South Africa Lancent 354; 471-476.

[6] Tess BH, Rodrigues LC, Newell ML, et al. Infant feeding and risk of mother-to-child transmission of HIV-1 in Sao Paulo State, Brazil. J Acquir Immune DeficSyndr Hum Retrovirol 1998; 19: 188-194.

[7] Haider R, Ashworth A, kabir 1. Huttley S. R. A; effect of community based peer counselor on exclusive breastfeeding practice in Dhaka, Bangladest, the lancet 356: 1643-1647, 2000 .

[8] Central Statistical Office (Zimbabwe) and Macro International Inc, 1995 Zimbabwe Demographic and Health Survey, 1994. Central Statistical Office and Macro International Inc. Calverton, Maryland.

[9] Friesen, P. et.al. 1998 infant feeding practices in Paupa New Guinea. Anm trop Paediatr. 18 (3): 209-215.

[10] Population References Bureau patterns in the developing world Measure communication, 1875.
[11] Gunnulaugsson, G \& J. Einarsdottir. 1993 Colostum and ideas about bad milk: a case study from Guniea Bissau Gunnlaugsson G(1), Einarsdóttir J. 36(3): 283-8.

[12] Hornnel A. et. Al. 1999, Breastfeeding patterns in exclusively breastfeeding infants I: a longitudinal prospective study in Upsala Sweden. Acta Paediatr. Int. Breastfeeding J; 88: 203211.

[13] Dewey KG, heing MJ, Nommsen LA maternal weight - 1080 patterns during prolonged lactation. AM J clin. Nutri 1993 58: 162-166.

[14] Central Statistical Office, 1998, Poverty Survey in Zimbabwe.

[15] Raphel, D \& F. Davis 1985. Only Mothers know: pattern of infant feeding bin traditional cultures.

[16] Maher, V. 1992, Breastfeeding and maternal depletion: natural law or cultural arrangement.

[17] Asfaw MM1, Argaw MD2, Kefene ZK3. Factors associated with exclusive breastfeeding practices in Debre Berhan District, Central Ethiopia: a cross sectional community based study.Int Breastfeed J. 2015 13; 10: 23. doi: 10.1186/s13006015-0049-2.

[18] WHO/Exclusivebreastfeedingwwwwho.int/nutritions/exclusive_breastfeeding/en/ accessed 2015 .

[19] Akhtaruzzaman M1, Hossain MA. Et al Attitude and Practices of Mothers on Breastfeeding Attended at a Tertiary Hospital in Bangladesh.Mymensingh Med J. 2015 Jul; 24(3): 480-5.

[20] Ministry of health and child welfare 1999, Department of Nutrition in Mimeo. Draft guidelines on infant feeding.

[21] T.O Lawoyin. Et al Factor associated with EBF in Ibadan, Nigeria. Journal of Human Lactation Vol: 17, No 4 pg 321325 (2001).

[22] Exclusive breastfeeding and associated factors in Enugu, Nigeria. Aghaji I.N et al. West African Journal of Medicine 2002 (Jan-Mar) 21 (1): 66-69. 